\title{
Standardization of MRI data and disability quantification in the post-EPIC era
}

\author{
Jagannadha Avasarala*
}

Department of Medicine, Division of Neurology, University of South Carolina School of Medicine, Greenville, South Carolina, USA

First published online 9 February 2017

Multiple sclerosis (MS) is a chronic immune-mediated neurological disease that is characterized by attacks on myelinated axons in the brain and spinal cord. The first drug approved by the Food and Drug Administration (FDA), Betaseron, significantly reduced the frequency of acute relapses in MS at two dose strengths (1.8 and $8 \mathrm{mIU}$, respectively) as compared to placebo. However, a discordant note was struck even in that first-ever study, as the Expanded Disability Status Scale (EDSS) shifted minimally from baseline in both the placebo and treatment arms.

The finding of Cree et al. ${ }^{1}$ (in the EPIC [expression/ genomics, proteomics, imaging, and clinical] study) that high-potency treatment protocols and no evidence of disease activity (NEDA) for two years in relapsing MS patients made no dent in long-term disability data followed prospectively over a 10 -year period is hardly surprising. What was unexpected was that the patient cohort that received escalated/aggressive therapies based on the NEDA criteria tended to have worse long-term outcomes compared to the group that received "platform therapy." It is possible that the NEDA criteria need to be applied for longer than two years to be effective in the long term. In MS, progression has proved to be a hard concept to quantify, and the pleotropic expression of MS makes it particularly challenging to measure all facets of this disease. Measurement of MS disease progression and treatment response is further complicated by individual patient heterogeneity, population variability in disease course, and the tempo of progression. Finally, no single drug in MS can reverse or arrest histopathological neurodegenerative endpoints. The failure of neuroprotective agents used in stroke, such as the glutamate

* Address for correspondence: Jagannadha Avasarala, Department of Medicine, Division of Neurology, University of South Carolina School of Medicine, Greenville Health System, 701 Grove Road, Greenville, South Carolina 29605, USA.

(Email: javasarala@ghs.org) receptor antagonists (Selfotel, Eliprodil, Aptiganel), antiinflammatory agents (Enlimomab, LeukArrest), ion-channel blockers (Nimodipine, Fosphenytoin, Maxipost), the free-radical scavengers (Citicoline), and other classes of drugs comes as no succor to MS clinicians who wish to arrest disability progression, albeit in a chronic disease state.

One of the glaring pitfalls in MS research is that magnetic resonance imaging (MRI) data are not collated identically across subjects, sites, and study populations to enable data pooling and analyses across populations. To circumvent the problem of standardization of data, the CombiRx Investigators Group ${ }^{2}$ developed an automated algorithm to weed out poor-quality images, as well as noncompliant protocol and patient motion artifacts. These cleaned-up data, obtained from a phase III clinical trial, were analyzed using the freely online-available open-source FreeSurfer software to measure global and regional cortical thickness. If MS researchers expand this concept to all FDA-approved drugs and pool data to analyze cortical thickness (focal, global, regional) and thalamic volumes between treated and control/placebo groups, a "ground truth" dataset can be accumulated to develop a gender-, ethnic-, and age-based database that could serve as the gold standard for future research groups invested in disability and for drug companies striving to arrest disability. To standardize data collection, as well as archiving and retrieval of MRI data in MS patients, a key organization such as the National Multiple Sclerosis Society ought to borrow a page from the Alzheimer's Disease Neuroimaging Initiative (2004) in leading efforts to build a national database that would ensure quality control, measure structural endpoints, and make longitudinal studies possible. One of the glaring challenges in following MS patients in the clinic is that no standardized MRI biomarkers that document disability from a radiological perspective exist. Unless this problem is resolved, studies will continue to churn 
out data that cannot be rationally interpreted given the nonexistent standardization.

Researchers in the MS field need to learn from the findings of the Optic Neuritis Treatment Trial, which showed that treatment with IV steroids followed by oral prednisone accelerated visual recovery but did not improve 12-month visual outcomes. ${ }^{3}$ This is because there are no drugs that regenerate ganglionic cells or restore the retinal nerve fiber layer lost in acute optic neuritis. Given this, how could the EPIC results be any different? By extension, how can axons that are transected/degenerated be restored? Conventional wisdom dictates that, unless the lesion load in MS is minimal and no new lesions appear while the disease is contained either by aggressive treatment of early MS or by natural regression of disease, such as occurs in the third trimester of pregnancy, disability will inevitably ensue despite treatment, because there are no drugs that address the fundamental neurodegenerative changes that drive MS. All MS drugs address the inflammatory component of the disease, but none have had any meaningful impact on the neurodegenerative component of the disease.

To date, all FDA-approved drugs in MS base their findings on control of relapses, which translates into blood/brain barrier stabilization. Typically, drugs are evaluated based on a stable EDSS with a 0.5- to 1.0-point variation that remains stable over a three-month period. Acute flare-up suppression by drugs in MS is quantifiable, but it is well known that acute relapses in MS correlates poorly with disability. Although disability data correlate with cortical thickness, ${ }^{4}$ thalamic atrophy, ${ }^{5}$ or deep gray matter iron deposition, ${ }^{6}$ no phase III drug trial has employed these endpoints as surrogate markers. However, in the context of the EPIC study findings and for disability data to be clinically meaningful, phase III studies in the future must incorporate these additional MRI criteria and analyze the effects of a drug on cortical thickness and thalamic volume changes.

Cortical thickness, thalamic atrophy, and deep gray matter involvement correlate with disability and serve as objective datapoints. It has been shown that EDSS score correlates with changes in cortical thickness of the bilateral sensorimotor cortex and bilateral insula. ${ }^{2}$ Baseline thalamic atrophy has also shown a significant correlation with deterioration in EDSS score over an eight-year period. ${ }^{5}$ Abnormal T2 hypointensity due to iron deposition most prevalent in the thalamus, putamen, and the caudate nucleus, as seen on susceptibility-weighted imaging, would be one other surrogate MRI marker that must be included in order to quantify disability. The validity of radiological criteria as objective surrogate markers has been demonstrated in another disorderdepression-characterized by hippocampal gray matter volume reduction. ${ }^{7}$ Restoration of cortical thickness is a tough therapeutic goal, but prevention of progression of cortical thinning might be a more tangible goal.

In summary, drugs that are approved for clinical use ought to attain newer and definable endpoints since stabilization of BBB and monitoring EDSS have proved to be insufficient, as reported in the EPIC study. While a solitary study cannot be used to relegate the indicators of disease progression that every study has used previously to develop new drugs, it is also relevant for the MS research community to meet the following criteria: (1) that MRI data in each phase III clinical trial be standardized and analyzed utilizing a template that cleans up the data; (2) that changes in cortical thickness and thalamic atrophy be introduced as secondary endpoints; (3) that MRI data archiving, retrieval, and analysis be standardized; and (4) that a universally accepted model of brain atrophy incorporating cortical thickness and thalamic volumes be defined for use in the clinic.

\section{Disclosures}

Jagannadha Avasarala hereby declares that he has nothing to disclose.

\section{REFERENCES:}

1. Cree BA, Gourraud PA, Oksenberg JA, et al. Long-term evolution of multiple sclerosis disability in the treatment era. Ann Neurol. 2016; 80(4): 499-510; Epub ahead of print Aug 13. https://www.ncbi.nlm. nih.gov/pmc/articles/PMC5105678/pdf/ANA-80-499.pdf. Accessed January 29, 2017.

2. Narayana PA, Govindarajan KA, Goel P, et al. Regional cortical thickness in relapsing remitting multiple sclerosis: a multicenter study. NeuroImage. 2012; 2: 120-131; https://www.ncbi. nlm.nih.gov/pmc/articles/PMC3777814/pdf/main.pdf. Accessed January 29, 2017.

3. Beck RW, Gal RL. Treatment of acute optic neuritis: a summary of findings from the optic neuritis treatment trial. Arch Ophthalmol. 2008; 126(7): 994-995; http://jamanetwork.com/journals/ jamaophthalmology/fullarticle/420682. Accessed January $29,2017$.

4. Sailer M, Fischl B, Salat D, et al. Focal thinning of the cerebral cortex in multiple sclerosis. Brain. 2003; 126(Pt 8): 1734-1744; Epub ahead of print Jun 4. https://academic.oup.com/brain/articlelookup/doi/10.1093/brain/awg175. Accessed January 29, 2017.

5. Rocca MA, Mesaros S, Pagani E, Sormani MP, Comi G, Filippi M. Thalamic damage and long-term progression of disability in multiple sclerosis. Radiology. 2010; 257(2): 463-469; Epub ahead of print Aug 19. http://pubs.rsna.org/doi/10.1148/radiol.10100326? url_ver=Z39.88-2003\&rfr_id=ori\%3Arid\%3Acrossref. org\&rfr_dat $=$ cr_pub\%3Dpubmed\&. Accessed January 29, 2017.

6. Bakshi R, Benedict RH, Bermel RA, et al. T2 hypointensity in the deep gray matter of patients with multiple sclerosis: a quantitative magnetic resonance imaging study. Arch Neurol. 2002; 59(1): 62-68; http://jamanetwork.com/journals/jamaneurology/ fullarticle/781179. Accessed January 29, 2017.

7. Videbech $\mathrm{P}$, Ravnkilde B. Hippocampal volume and depression: a meta-analysis of MRI studies. Am J Psychiatry. 2004; 161(11): 1957-1966; http://ajp.psychiatryonline.org/doi/pdf/10.1176/appi. ajp.161.11.1957. Accessed January 29, 2017. 